Palabras clave: Agresión, Trastorno de Estrés

Postraumático, Dependencia alcohólica.

\title{
Agresión en el Trastorno de Estrés Postraumático comórbido con Dependencia Alcohólica
}

\author{
Zoran Zoričić, MD \\ Danijel Buljan, MD \\ Vlatko Thaller, MD, PhD \\ Dalibor Karlović, MD
}

Departamento Universitario de Psiquiatría, Hospital Universitario "Sestre milosdnice", Zagreb

CROACIA

\begin{abstract}
RESUMEN - El objetivo de este estudio es el de investigar la dependencia del alcohol en soldados profesionales de combate con Trastorno de Estrés Postraumático (PTSD) relacionado con el incremento de la agresión. Se entrevistaron dos grupos de soldados de combate profesionales, utilizando entrevistas clínicas estructuradas basadas en el criterio DSM IV para PTSD crónico $(\mathrm{N}=40)$, y PTSD coexistente con dependencia al alcohol $(\mathrm{N}=40)$. La escala de ratios para la agresión A-87 se utilizó para explorar el nivel de agresión entre estos grupos. Los soldados profesionales de combate con PTSD comórbido con una dependencia al alcohol, mostraron mayores niveles de agresión verbal que aquellos soldados que presentaban únicamente PTSD, (54,97+- 8,62 vs 49,47 +- 12,76; $\mathrm{t}=2,19 ; \mathrm{p}<0,05)$, así como conductas manifiestas de agresión física $(41,75+-9.80$ vs 35,62 +- 10,80; $\mathrm{t}=2,96 ; \mathrm{p}<0,05)$. La comorbilidad con dependencia al alcohol podría jugar un papel en cuanto a los niveles de agresión en las experiencias de PTSD crónicos especialmente al presentarse la agresión.
\end{abstract}

\section{Introducción}

El trastorno de estrés post-traumático (PTSD) está a menudo asociado a otras perturbaciones psiquiátricas (Keane, Wolfe 1990). Hay una gran literatura acerca de la prevalencia de dependencia del alcohol en veteranos de combate, y se ha informado que el $60-80 \%$ de los pacientes con PTSD realizaban concomitantemente un abuso o adicción a drogas o al alcohol (Kofoed et al. 1993, Thaller et al. 1997, Branchley et al. 1984), mostrando como la dependencia al alcohol es el mas común de los codiagnósticos del PTSD.

Se han dado varias explicaciones acerca de la relación entre el PTSD y el alcoholismo. Los modelos de "automedicación" (Khantzan 1985) y la "reducción de tensión” (Lehman et al. 1989) postulan que el 
PTSD se desarrolla en primer lugar, y el abuso del alcohol aparece en segundo lugar con el fin de reducir la tensión. Sin embargo existe una evidencia que muestra como el alcoholismo está relacionado con el incremento de la agresión (Conklin 1992).

Además, un síntoma de grupo ("cluster") denominado "hiper-arousal", en los Criterios para el Diagnóstico del PTSD en el Manual Estadístico y de Diagnóstico de Trastornos Mentales, en su cuarta edición (DSM IV) está relacionado con la agresión (Asociación Psiquiátrica Americana 1994). Según esto, el objetivo de este estudio es investigar si la dependencia del alcohol en soldados profesionales croatas con PTSD crónico está relacionada con un incremento de la agresión.

\section{Metodología}

\section{Sujetos}

El grupo de estudio de veteranos de guerra con PTSD relacionado con el combate y comórbido con una dependencia al alcohol consistió en 40 hombres, de edad entre 2456 años (Mediana: 36,8). El grupo de control consistía en 40 hombres veteranos de guerra, de edad entre 23-54 años (Mediana: 35,2) con solo un PTSD crónico relacionado con el combate. Este grupo se eligió porque se parecía al grupo de dependencia al Alcohol comórbido con PTSD en cuanto a factores demográficos, incluyendo edad, etnia y clase socioeconómica.

Ninguno de los pacientes investigados tenía algún otro trastorno psiquiátrico o problema médico, y todos dieron consentimiento para su participación en el estudio. Todos los pacientes habían consumido drogas de tipo psicofarmacológico con anterio- ridad a este estudio, en su mayoría antidepresivos y ansiolíticos.

\section{Exámen médico}

El diagnóstico de PTSD actual o la dependencia al Alcohol se realizó de acuerdo a unas entrevistas clínicas estructuradas basadas en los criterios DSM IV (Asociación Psiquiátrica Americana). Dos psiquiatras realizaron esta parte de la evaluación, cada uno de ellos examinaba de forma independiente a todos los individuos. El acuerdo que existía entre los dos psiquiatras era alto, 0,97. Un psicólogo clínico realizó la entrevista para el PTSD de Watson, basado en criterios DSM-III-R para medir reacciones de estrés post-traumático (Watson et al. 1991). El acuerdo entre el criterio psiquiátrico y psicológico fue de un 0,95.

Medimos el nivel de agresión a través de la escala de ratios A-87 de Zuzul. Esta escala consiste en cinco subescalas: Agresión manifiesta verbalmente (VMA), agresión manifestada físicamente (FMA), agresión indirecta (IA), agresión latente verbalmente (VLA), y agresión latente físicamente (FLA). La evaluación de las características psicométricas de este instrumento en una muestra croata confirmaron su alta validez (Kerestes, Zuzul 1992).

\section{Análisis estadístico}

La distribución normal se valoró para todas las medidas y para cada grupo utilizando el test Kolmogorov-Smirinov. Los datos de VMA, FMA, IA, VLA y FLA se analizaron utilizando un t-test para muestras independientes. Las estadísticas se hicieron con software SPSS (SPSS para Windows 8.0, SPSS., Chicago, IL, USA). 


\section{Resultados}

No hubo diferencias estadísticamente significantes $(\mathrm{t}=0,99 ; \mathrm{p}=0,32)$ en cuanto a las edades entre PTSD crónicos (Media +- SD; 35,2 +- 6,2) versus PTSD crónicos unido a la dependencia del alcohol (Media +- SD; 36,8 +- 7,1). El 62,5\% de los pacientes con PTSD crónico comórbido con dependencia al alcohol, y el 67,5\% de los pacientes con PTSD crónico poseían un nivel alto de formación, y no había una diferencia significativa entre estos dos grupos en cuanto a la educación (Chi-Cuadrado $=22,16 ; \mathrm{p}=0,98)$. En cuanto al estado civil, un $55 \%$ de los pacientes con un PTSD crónico comórbido con una dependencia al alcohol, no estaban casados y el $60 \%$ de los pacientes con solo PTSD (Chi-Cuadrado $=20,83 ; \mathrm{p}=0,99)$ tampoco lo estaban.

El nivel de agresión basado en la escala de ratios de agresión A-87 entre los combatientes con PTSD crónico comórbido con dependencia al alcohol, era mayor (Media +- SD; 250,75 +- 38,42) que en el grupo de control con PTSD crónico (Media +- SD; $238,60+-40,19)$ aunque no significativo estadisticamente $(\mathrm{t}=0,01 ; \mathrm{df}=39 ; \mathrm{p}=$ 0,91).
El nivel de agresión manifestado verbalmente, así como el nivel de agresión física resultaron ser estadísticamente mas significativos entre los combatientes con PTSD comórbido con una dependencia del alcohol que en el grupo de solo combatientes con PTSD (tabla I).

No hubo diferencias significativas entre el grupo de estudio y el grupo de control en cuanto a las escalas de agresión latente verbal, latente física y agresión indirecta (tabla I).

\section{Discusión}

Los resultados sugieren un incremento en el nivel de agresión manifestada verbalmente así como la manifestada físicamente entre los combatientes con PTSD comórbido con una dependencia al alcohol, como oposición a los PTSD crónicos sin dependencia al alcohol. La dependencia al alcohol y un nivel incrementado de comportamiento agresivo con manifestaciones físicas o verbales en los soldados con PTSD no se relacionan con la edad, con la educación o con el estado civil, al contrario de lo que ocurre con los soldados con PTSD y sin dependencia al alcohol.

\section{Tabla I}

Comparación entre el grupo de soldados con PTSD relacionado con el combate y el grupo de soldados con PTSD relacionado con el combate y con dependencia alcohólica en relación con las puntuaciones de VMA (agresión verbal manifiesta), FMA (agresión física manifiesta), IA (Agresión indirecta), VLA (Agresión verbal latente), FLA (Agresión física latente) y A (Agresión Total)

\begin{tabular}{lcccr} 
& PTSP $($ Mdia \pm SD) & PTSP \pm Alc. $($ Media \pm SD) & $\mathrm{t}$ & $\mathrm{p}$ \\
\hline VL & $55,82 \pm 12,26$ & $55,85 \pm 7,37$ & 0,01 & 0,91 \\
FL & $53,40 \pm 14,02$ & $53,00 \pm 11,39$ & 0,15 & 0,87 \\
IN & $44,58 \pm 9,81$ & $45,17 \pm 8,21$ & 0,31 & 0,75 \\
FM & $35,62 \pm 10,80$ & $41,75 \pm 9,80$ & 2,96 & $<0,05$ \\
VM & $49,47 \pm 12,76$ & $54,97 \pm 8,62$ & 2,19 & $<0,05$ \\
A & $238,60 \pm 40,19$ & $250,75 \pm 38,42$ & 1,45 & 0,15 \\
\hline
\end{tabular}


El comportamiento agresivo entre los individuos con PTSD es uno de los factores mas comunes presentes en los problemas conyugales, exactamente el mismo que con la dependencia del alcohol (Byrne, Riggis 1996). La dependencia al alcohol está asociada con muchas otras actividades criminales, especialmente con el comportamiento antisocial, con el suicida y con el homicida (Buljan et al. 1996)

El comportamiento agresivo es uno de los síntomas que pertenecen al grupo de síntomas propios del PTSD (Asociación Psiquiátrica Americana 1994). Existen otras opiniones que afirman que el comportamiento agresivo se estimula especialmente a través del abuso del alcohol. De acuerdo con esto, la dependencia al alcohol que es uno de los mas comunes diagnósticos psiquiátricos comórbidos con el PTSD, constituye la causa mas seria de los comportamientos agresivos en los pacientes con PTSD. En otras investigaciones relacionadas con la dependencia al alcohol en enfermos con PTSD el alcohol serviría para reducir la tensión utilizándose a modo de automedicación (Khantzan 1995, Lehman et al 1989). Pero teniendo en cuenta nuestros hallazgos, no existe explicación para una hipótesis de "automedicación", al menos en lo referente a los comportamientos agresivos de los pacientes con PTSD. Además existe una gran evidencia de que la dependencia al alcohol altera el metabolismo de la serotonina (Buljan et al. 1996), y la serotonina, por otra parte es uno de los neurotrasmisores mas importantes para el control de la agresión (Jacobs 1991). Otros estudios sugieren que la serotonina es un neurotransmisor que regula el humor, el "arousal", el sueño, y también el comportamiento agresivo (Soutwich et al. 1997). La actividad monoaminoxidasa $\mathrm{B}$ en las plaquetas (MAO-B) ha sido propuesto como un indicador del sistema serotoninérgico (Orleand, Shaskan 1983), y una baja actividad plaqueta- ria (MAO-B) ha sido propuesta como factor de riesgo psicopatológico en diversas condiciones psicopatológicas, como la dependencia del alcohol, el comportamiento agresivo y antisocial, las conductas de tipo psicopático y la esquizofrenia (Liewovitz et al. 1990). Davidson et al. (1985) encontraron una actividad MAO-B significativamente mas baja en pacientes con PTSD. Cuando los pacientes con PTSD se dividían entre los que poseían antecedentes en abuso del alcohol y los que no los poseían, únicamente el grupo anterior difería significativamente del grupo de control (Davidson et al. 1985).

Pero también las explicaciones psicodinámicas acerca del consumo de alcohol servirían para explicar nuestros resultados. Así, el alcohol como supresor del Superego que es (moral, normas sociales) (Blane, Leonard 1987), en soldados con PTSD comórbido con una dependencia al alcohol, reduciría la retención de la agresión en el nivel latente, y colaboraría a expresar la agresión en el nivel manifiesto, al contrario de lo que ocurre entre los soldados con PTSD y sin dependencia alguna al alcohol.

En estos hallazgos biológicos y psicológicos, podemos encontrar la explicación de nuestros resultados referntes a como los individuoscon PTSD y con dependencia al alcohol muestran un incremento en sus niveles de agresión manifiesta verbal y física a diferencia de lo que ocurre en los individuos con PTSD sin dependencia al alcohol. Serían necesarios estudios más profundos con el fin de comparar la influencia que pueda tener la dependencia del alcohol sobre síntomas específicos del PTSD, especialmente en los síntomas intrusivos, evitativos e "hiperarousales", así como el poder comparar el comportamiento agresivo y la dependencia del alcohol en pacientes PTSD con sus niveles de serotonina y de actividad MAO-B. 


\section{Bibliografía}

AMERICAN PSYCHIATRIC ASSOCIATION. Diagnostic and statistic manual of mental disorders, IV edition. Washington DC, APA, 1994.

BLANE, H., LEONARD, K.E. Psychologycal theories of drinking and alcoholism, Guilford, New York, 1987.

BRANCHEY, L., DAVIS, W., LIEBER, C.S. Alcoholism in Vietnam and Korea veterans: a long-term followup. Alcohol Clin. Exp. Res., 8, 572-575, 1984.

BULJAN, D., BRZOVIĆ, Z., THALLER, V., BREITENFELD, D., MARUŠIĆ, S. Neurotransmitter changes in alcoholism and withdrawal syndrome (Neurobiological test of alcoholism). Coll. Antropol., 1, 175-182, 1996.

BYRNE, C.A., RIGGS, D.S. The cycle of trauma; relationship aggression in male Vietnam veterans with symptoms of posttraumatic stress disorders. Violence \& Victims, 11, 213-225, 1996.

CONKLIN, J. Criminology, New York, Mac Millan Publising Co., 1992.

DAVISON, J., LIPPER, S., KILTS, C.D., MAHORNEY, S., HAMMETT, E. Platelet MAO activity in posttraumatic stress disorder. Am. J. Psychiatry, 142, 1341-1343, 1985.

JACOBS, B.L. Serotonin and behavior: emphasis on motor control. J. Clin. Psychiatry, 12, 17-23, 1991.

KEANE, T.M., WOLFE, J. Comorbidity in posttraumatic stress disorder: an analysis of community and clinical studies. J. App. Soc. Psychiatry, 20, 1776-1788, 1990.

KERESTEŠ, G., ŽUŽUL, M. Priručnik za primjenu Upitnika za mjerenje agresivnosti, Jastrebarsko, Naklada Slap, 1992.

KHANTZAN, E.J. The self-medication hypothesis of addictive disorders: focus on heroin and cocaine dependence. Am. J. Psychiatry, 142, 1259-1264, 1985.
KOFOED, L., FRIERDMAN, M.J., PECK, R. Alcoholism and drug abuse in patients with PTSD. Psychiatric $Q$., 64, 151-171, 1993.

LEHMAN, A.F., MYERS, P., CORTY, E. Assessment and classification of patients with psychometric and substance abuse syndromes. Hospital and Community Psychiatry, 40, 1019-1025, 1989.

LIEWOVITZ, M.R., HOLLANDER, E., SCHNEIER, F., CAMPEAS, R., WELKOWITZ, L., HATTERER, J. Reversible and irreversible monoamine oxidase inhibitors in other psychiatric disorders. Acta Psychiatr. Scand., 82, 29-34, 1990.

ORELAND, L., SHASKAN, E.G. Some rationale behind the use of monoamine oxidase activity as a biological marker. Trends in Pharmacological Sciences, 4, 339341, 1983.

SOUTHWICH, S.M., KRYSTAL, J.H., BREMNER, J.D., MORGAN, C.A., NICOLAN, A.L., NAGY, L.M. Noradrenergic and serotoninergic function in posttraumatic stress disorder, Arch. Gen. Psychiatry., 54, 749-758, 1997.

THALLER, V., BREITENFELD, D., BULJAN, D. Alcohol consumption Among Croatian Combat veterans. Eur. J. Psychiat., 11, 43-50, 1997.

WATSON, C.G., JUBA, M.P., MANIFOLD, U., KUCALA, T., ANDERSON, P.D. The PTSD interview: rationale, description, reliability, and validity of a DSM-III based technique, J. Clin. Psychol., 47, 179-186, 1991.

Dirección para correspondencia:

Zoran Zoričić

Departamento Universitario de Psiquiatría

Hospital Universitario "Sestre milosdnice"

Vinogradska, 29

10000 Zagreb

CROACIA 